\title{
Raynaud Phenomenon: Diagnosis and Management
}

\author{
Fitriani Fitriani ${ }^{1 *}$, Citra Tresna Murti ${ }^{1}$, Soenarto $\mathrm{K}^{1}$ \\ ${ }^{1}$ Dermatology and Venereology Department, Faculty of Medicine Universitas Sriwijaya \\ \#Corresponding author E-mail: fitrianihasan@ymail.com
}

Received : January $20^{\text {th }} 2020$

Accepted : March $28^{\text {th }} 2020$

\begin{abstract}
Raynaud's phenomenon (RP) is a chronic episodic attack of digital ischemia provoked by exposure to cold or emotional stres. This phenomenon affects $3-5 \%$ of the population, with female ratio more than men and is categorized into a primary and secondary form. Primary form of RP is idiopathic, meanwhile the secondary form is associated with underlying diseases and other condition such as connective tissue disease, obstructive arterial disease, neurologic disorders, drugs and toxin, hyperviscosity disorders, infections, endocrine disturbance, neoplasms, and occupation or environmental exposure. Until today, to classified and build the diagnosis of RP is still a clinical challenge. Therefore, a careful history and a physical examination, together with laboratory tests and nailfold capillaroscopy are mandatory. A combination of conservative measures and medications can help in the management of RP. The approach to the RP patients requires therefore a coordinated care of specialists including dermatology.
\end{abstract}

Keywords: Raynaud's phenomenon, diagnosis, management

\section{Introduction}

Raynaud's phenomenon (RP) is an episodic digital ischemia that occurs due to cold stimulus or emotional stress. This phenomenon is characterized by a series of changes in the color of the fingers called triphasic color, which is white (pallor), blue (cyanosis), and red (rubor), accompanied by numbness and ended with episodes of pain. ${ }^{1}$

Raynaud's phenomenon is divided into primary and secondary RP. Raynaud's phenomenon is more likely to be used to refer to secondary RP, whereas primary RP is often referred to as Raynaud's disease. The primary Raynaud phenomenon is idiopathic, whereas secondary RP arises because of an underlying disease or condition. In its journey, the transitions from primary RP to secondary RP can occur. ${ }^{2}$

The prevalence of RP varies among different geographical areas, depending on climate, ethnicity, and diagnostic methods. ${ }^{3}$ The prevalence of this phenomenon in the general 
population is around $3-5 \%,{ }^{4}$ with primary RP more often occur than secondary RP, and more often occur in women than men. ${ }^{5}$ The highest prevalence of secondary RP is in patients with systemic sclerosis or scleroderma and mixed connective tissue disease (MCTD), reaching 90\% or more. In addition, systemic rheumatic diseases such as systemic lupus erythematosus (SLE), primary Sjögren's syndrome, rheumatoid arthritis (RA), and polymyositis can also cause secondary RP. 6

The pathophysiology of vasospasm as a cause of RP is complex and not yet fully understood. Abnormalities in the central control mechanism of thermoregulation, vascular, neural, intravascular factors, and other factors such as hormones and genetics play a role in the occurrence of RP. ${ }^{7}$

A careful history and physical examination are important in the evaluation of RP patients. The main thing in RP diagnosis is, knowing that RP is dividing into the primary or secondary form. A clear description of an ischemic attack will help identify signs and symptoms that indicate a secondary cause. ${ }^{8}$ In addition, severe RP complications such as ulcers and gangrene, generally occur in secondary RP. Gold standard examination for RP is nail fold capillaroscopy.

The management of RP depends on the frequency, severity of attacks, and complications of ischemia. Primary Raynaud's phenomenon is generally easy to control with lifestyle changes, while secondary RP needs to be treated according to the underlying disease or condition, which makes comprehensive management is needed. Medication is given if RP attacks are prolonged or fail to be treated conservatively. Calcium channel blockers (CCB) are by far the most widely used and effective way to treat RP. ${ }^{9}$

\section{Classification}

Raynaud's phenomenon is divided into primary and secondary RP. The difference between the two types of RP is based on the presence or absence of underlying causes or diseases. Primary Raynaud's phenomenon or Raynaud's disease arises without underlying or idiopathic causes. While secondary RP or also called Raynaud's syndrome or phenomenon, arises due to various causes. ${ }^{1}$ Primary Raynaud's phenomenon generally first arises in adolescence in the range of 15-30 years, while secondary RP arises at age > 25 years. ${ }^{10,11}$

About 1-3\% of primary RP patients per year develop into secondary RP, especially in patients with systemic sclerosis. This is presumably because in addition to inaccuracies in the 
ISSN 25980580

initial classification as primary RP, it can also be caused by RP in the early phase of connective tissue disease that can manifest clinically similar to primary RP. ${ }^{2}$

\section{Etiology And Pathogenesis}

Raynaud was the first person who reveal the initial etiology of RP. He mentioned that $\mathrm{RP}$ is a local asphyxia of the extremities caused by disorders of the central nervous system (CNS), which then affected the innervation of blood vessels. Lewis and Pickering, however, estimate that RP is a local defect caused by digital arterial spasm and not a secondary result of CNS damage. ${ }^{12}$ Raynaud's phenomenon proved to be provoked not only by local cold exposure but also by stress, which implies that both local and central mechanisms are involved. ${ }^{7}$

Risk factors for primary RP include genetic factors, smoking habits, and a history of migraine. ${ }^{3}$ While secondary RP can be caused by several diseases or conditions as can be seen in table 1.

Table 1. Disease and Condition Causing Secondary RP

\begin{tabular}{|c|c|}
\hline DISEASE/CONDITION & Type \\
\hline \multirow[t]{7}{*}{ Connective Tissue disease } & Scleroderma \\
\hline & SLE \\
\hline & Dermatomyositis and polimiositis \\
\hline & Undifferentiated connective tissue disease \\
\hline & Sjögren's Syndrome \\
\hline & RA \\
\hline & Multiple myeloma \\
\hline \multirow[t]{4}{*}{ Arterial obstruction disease } & Atherosclerosis \\
\hline & Thromboangiitis obliterans (TAO) \\
\hline & Thromboembolism \\
\hline & Thoracic outlet syndrome \\
\hline \multirow[t]{4}{*}{ Neurologic disorder } & Carpal tunnel syndrome \\
\hline & Hemiplegia \\
\hline & Polyomyelitis \\
\hline & Syringomyelia \\
\hline \multirow[t]{8}{*}{ Drugs } & Propranolol \\
\hline & Preparat ergot \\
\hline & Methysergide \\
\hline & Obat intra-arteri \\
\hline & Zat psikoaktif \\
\hline & Siklosporin \\
\hline & Kontrasepsi oral \\
\hline & Agen kemoterapi (bleomycin, vinblastine) \\
\hline \multirow[t]{2}{*}{ Work } & Jobs that require prolonged cold temperatures to the extremities \\
\hline & Work that uses a vibrating device \\
\hline \multirow[t]{3}{*}{ Hyperviscosity disorder } & Cryoglobulinemia \\
\hline & Macroglobulins \\
\hline & Cold agglutinin \\
\hline
\end{tabular}


Connective tissue disease is the most common cause of secondary RP. A range of 8090\% of scleroderma patients experience persistent RP and/or vasospasm. Raynaud's phenomenon can be the only clinical manifestation that has existed for years. ${ }^{9}$

According to Herrick et al, there are at least four things that underlie the pathogenesis of RP, including (1) vascular, (2) neural, (3) intravascular, and (4) other factors. ${ }^{7}$

\section{Vascular}

Vascular abnormalities in RP consist of (1) vasodilation disorders, (2) increase in vasoconstriction, and (3) abnormalities or changes in microvascular structures and digital arteries. The primary Raynaud phenomenon is caused by impaired vascular and/or nerve function, and does not risk irreversible tissue damage, while secondary RP is caused by changes in vascular structure and results in tissue damage such as digital ulceration, scarring, or gangrene. $^{7}$

Changes in vascular function in the RP are primarily on the endothelium which plays a role in regulating vascular tone by producing a number of vasodilator mediators such as nitric oxide (NO), and vasoconstrictor mediators such as endothelin-1, and angiotensin. ${ }^{6,7}$ Activation and/or endothelial damage will disturb the balance between vasodilation and vasoconstriction which results in less vasodilator production or effectiveness and/or excessive vasoconstrictor production. ${ }^{6}$ Disorders of endothelial cells can cause vasoconstriction even without damage to vascular structures. ${ }^{7}$

\section{Neural}

Neural factors in the pathogenesis of RP play a role in conditions caused by direct disorders of the sympathetic nervous system, vasodilation disorders, excessive vasoconstriction, and central mechanisms. ${ }^{7,9}$

One of the neurotransmitters vasodilatation that plays a role is calcitonin-gene related peptide, with decreased immunoreactivity in the finger skin of RP patients. In addition, there is a disruption in the function of alpha- 2 adrenergic receptors that play a role in regulating 
vascular tone. Sensitivity to cold is thought to be caused by alpha-2C adrenergic receptors, which move from the golgi compartment to the cell surface in response to cold. The relocation of alpha-2C adrenergic receptors is induced by Rho kinase, which is stimulated by reactive oxygen species (ROS) produced in smooth muscle cells in response to cold. Arteriol vasoconstriction is also associated with increased activity of tyrosine kinase protein and tyrosine phosphorylation in both primary and secondary RP. In addition, nonadrenergic mechanisms, such as the neuropeptide Y pathway, can also contribute to skin vasoconstriction. ${ }^{7}$ Although the central mechanism alone cannot fully explain the process in RP, many RP patients experienced vasospasm induced by stress. This shows the influence of the CNS. According to research conducted by Freedman et al, the range of one third of primary RP attacks is caused by emotional stress, whereas secondary RP is less affected by stress. Several studies investigating the effects of emotional stress on vascular tone and digital perfusion in RP patients have produced mixed conclusions, and none can explain the role of the sympathetic nervous system in the pathogenesis of this disorder. However, studies of cardiovascular responses that are triggered by stressors in RP patients show impaired ability to deal with repetitive stress stimuli, which shows changes in CNS control of the cardiovascular system. ${ }^{12}$

\section{Intravascular}

Several factors in circulation have been shown to be involved in the pathogenesis and exacerbation of RP, particularly when associated with secondary RP, especially systemic sclerosis. The roles of platelet activation, fibrinolysis damage, and oxidative stress have been reported. Although the role of these factors is not fully understood, these intravascular factors can exacerbate digital vasospasm by reducing blood flow in the microvasculature. ${ }^{12}$

\section{Other Factor}

Other factors that play a role in the pathogenesis of RP include the estrogen hormone, and genetic factors. The role of estrogen in vascular disorders has long been suspected, because women dominate blood vessel disorders such as primary pulmonary hypertension and migraine. In addition, a higher prevalence of RP in women, and an increase in RP in the phase between menarche and menopause proves that differences in sex and hormonal factors are important. ${ }^{7,8}$ The effects of estrogen on vascular tone are not fully understood, so recommendations for estrogen use or restriction in RP patients seem still too early. ${ }^{12}$ 
ISSN 25980580

A study using whole genome linkage analysis in family members with RP identified 3 potential gene candidates, namely the beta acetylcholine receptor muscle subunit, serotonin $1 \mathrm{~B}$ and $1 \mathrm{E}$ receptors. Other studies report a higher prevalence of migraines in primary RP patients, with migraine symptoms more evident in patients who have a family history of RP. These findings indicate that primary RP is part of a broader vascular tone disorder with genetic predisposition. ${ }^{12}$ About $30-50 \%$ of primary RP patients has first-degree relatives who also experience primary RP. ${ }^{10}$ In addition, genetic factors are also involved in the pathogenesis of secondary RP especially systemic sclerosis. ${ }^{12}$

\section{Clinical Manifestation}

Patients with RP commonly complain about episodic attacks due to cold exposure and emotional stimulation in the form of triphasic color changes that are clearly demarcated, start with white (pallor) due to ischemia, then blue (cyanosis) due to deoxygenation, and finally red (hyperemia) due to reperfusion. Complete changes in these three colors are rarely seen, most of which only show pale fingers accompanied by numbness (figure 1). ${ }^{2}$ Most experts believe that changes in white and blue biphasic color are sufficient for clinical features of RP, with the pallor phase being the most important of the 2 other color change phase. ${ }^{1,10}$

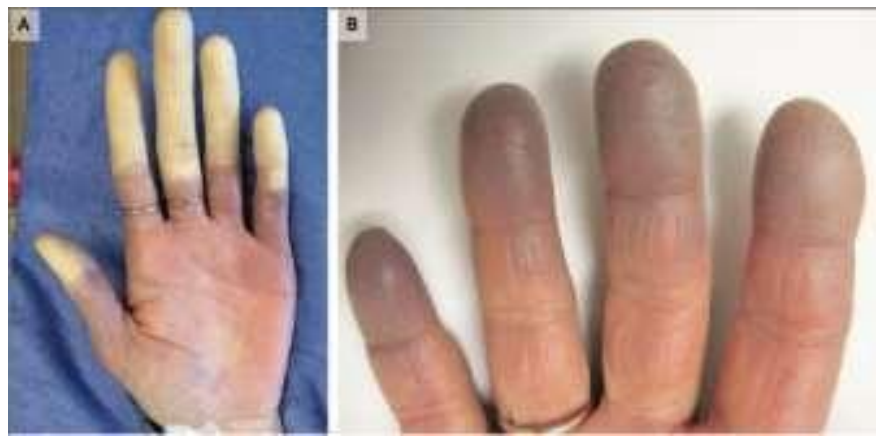

Figure 1. Clinical manifestations of RP. A. Pallor phase. B. Cyanosis phase ${ }^{10}$

The most commonly affected body parts by RP are the fingers and toes. Raynaud's phenomenon however can also occur on the tongue (figure 2), nose, ears, or nipples. ${ }^{3}$ During an attack, one or more fingers become numb. When warmed, the finger becomes bright red, and can be accompanied by throbbing pain. Attacks can last for several minutes, up to several 
hours. ${ }^{9,13}$ The frequency of attacks on primary RP is $<5$ times per day, while secondary RP attacks can occur 5 to 10 times per day. ${ }^{11}$

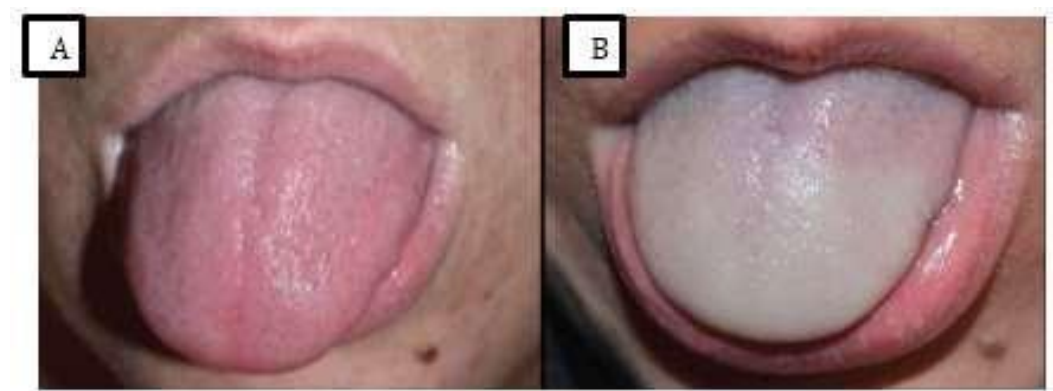

Figure 2. Clinical Manifestation of RP on Tongue. A. Normal tongue, B. Tongue during RP ischemic attack. ${ }^{14}$

In primary RP, vasospasm attacks are generally symmetrical, but often only affect a portion of the finger, and without thumb involvement. Attacks on primary RP also quickly improve with warmth, and no ischemic injury is seen. ${ }^{10,15}$

In secondary RP, asymmetrical vasospasm attacks occur with thumb involvement. The attacks are intense, frequent, accompanied by pain in the ischemic phase, edema, and are at risk of ischemic injuries such as ulcers on the finger pads or around the nail bed, or gangrene. The ulcer will then heal slowly, leaving a characteristic scar tissue in the form of a point called digital pitting or pitting scar (figure 3). ${ }^{2}$

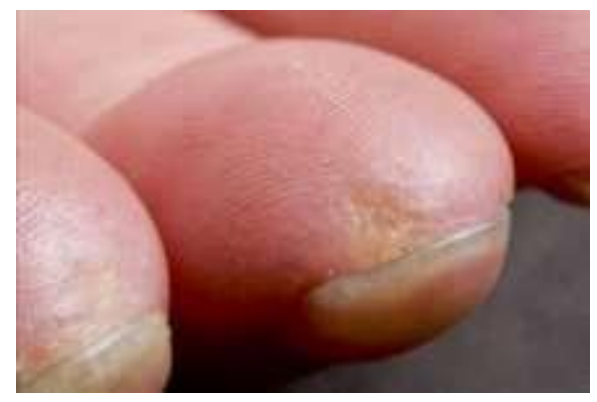

Figure 3. Pitting scar on secondary RP. ${ }^{2}$

In addition, nail dystrophy, skin atrophy, calcinosis, telangiectasia, sclerodactile, and hair loss on the dorsal surface of the fingers in secondary RP patients caused by persistent vascular abnormalities can also occur (figures 4). ${ }^{2,9,15}$ 


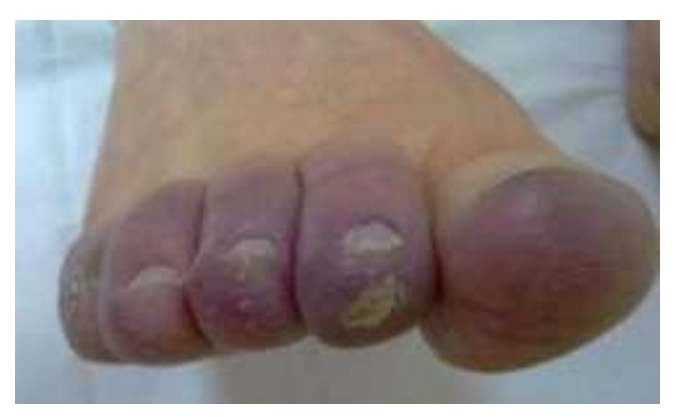

ISSN 25980580

Figure 4. Persistent vascular abnormalities in secondary RP. Damage to the nails and ulcers on the finger pads. ${ }^{15}$

In secondary RP caused by TAO, the pulse in both arms can be asymmetrical, so the overall pulse and blood pressure of both arms must be checked. ${ }^{9,16}$ In addition, it is necessary to look for muscle weakness, joint pain, dry eyes and mouth or sicca symptoms, as well as complaints of heartburn that indicate esophageal dysmotility to see possible secondary causes of connective tissue disease, especially scleroderma. ${ }^{2,8}$

\section{Additional Examination}

Examination for RP consist of Allen tests, laboratory tests, and non-invasive tests. ${ }^{9}$

\section{Allen Test}

The Allen test is useful for assessing the function of arteries and capillaries of the hand. In this test, the radial and ulnar arteries are simultaneously compressed by the examiner's thumb, while the patient clenches his fists for 30 seconds. After that, the patient asked to open his hands, and the patient's hands can be seen pale. Pressure is released from the ulnar artery but the radial artery is maintained. If the hand's color returns within 5-15 seconds, arterial function is considered normal. Abnormal filling indicates structural abnormalities of the microcirculation and increases suspicion of secondary RP., ${ }^{9,17}$

\section{Laboratory Test}

In RP patients, laboratory tests that need to be done are complete blood tests, erythrocyte sedimentation rates, and ANA tests. ${ }^{2,9}$ ANA tests help identify RP patients who are at risk for connective tissue disease. This test should be a routine examination in secondary RP patients because $>90 \%$ of secondary RP is due to connective tissue disease. Additional laboratory tests can be carried out according to the findings on history taking and physical examination. ${ }^{2}$

\section{Non-invasive test}


Some non-invasive tests that can be performed on RP are capillaroscopy, infrared termography (IRT), Doppler ultrasound, finger systolic pressure measurement, and plethysmography. ${ }^{2}$

Capillaroscopy is the gold standard examination for RP. This examination aims to see the morphology of the capillaries in the nail fold dermis papillae. The image of a normal distal capillary row is hairpin-shaped loops that are parallel to the finger axis. Mild capillary abnormalities in the form of twisted capillaries can be seen in primary RP patients. ${ }^{2}$

In secondary RP particularly due to scleroderma, there are typical capillaryoscopic features such as capillary dilation, decreased capillary count, bleeding, and neoangiogenesis. ${ }^{2}$

IRT cameras measure infrared emissions to estimate the surface temperature of an object. This tool is used clinically to assess disease progression and therapeutic response. Changes in skin temperature due to perfusion and blood flow can be detected by IRT, so that indirect measurements can be made of skin microvascular function. ${ }^{2}$

Laser Doppler technique, arterial Doppler ultrasound, finger systolic pressure, and plethysmography are RP diagnosis techniques that are used in research and are not used routinely for clinical assessment. ${ }^{2}$

\section{Diagnosis}

The diagnosis of RP is built on the basis of history, clinical manifestations, and additional examination, all of which aim to identify whether the RP is primary or secondary. ${ }^{9}$

There are diagnostic criteria for primary RP, including (1) vasospastic attacks precipitated with cold exposure or emotional stimulation, (2) bilateral limb involvement, (3) examination of blood vessels, peripheral pulses, and capillary microscopy of normal nail fold, (4) absence of gangrene or, if present, limited to fingertip skin, (5) no underlying disease, drug, or occupational exposure that causes a vasospasm attack, (6) a negative ANA test, (7) normal erythrocyte sedimentation rate, and (8) history symptoms for at least two years. ${ }^{9}$ A summary of the evaluation of RP can be seen in Figure 7.

\section{Differential Diagnosis}

Some skin diseases related to temperature sensitivity can mimic RP, such as frostbite, chilblains, lupus chilblains, acrosianosis, livedo reticularis, and erythromelalgia. This disease 
is distinguished based on the cause, skin character and tissue changes, and the course of the disease. $^{2}$

Frostbite is an ischemic condition due to extreme and prolonged cold exposure. Frostbite and RP are caused by temperature sensitivity, which is mainly concerning the extremities. Patients with RP are at risk of suffering from frostbite, and conversely frostbite can increase the risk of RP. Raynaud's phenomenon improves with heating, and generally in mild to moderate cases is limited to white, blue or red. Severe finger ischemia in secondary RP can resemble frostbite images, with digital violaceous, multiple, and necrotic ulcers. ${ }^{2}$

Chilblains are cold induced vasculopathies that are more commonly seen in humid climates and are not associated with systemic diseases. This condition mainly affects the hands and feet with bilateral symmetrical distribution and is more common in young women. Inflammatory lesions typically in the form of purplish red macules can be seen at the distal end of the affected area, and usually arise within a few hours after cold exposure. Burning, pain, and/or pruritus, swelling of small joints in the area involved, and ulceration, often accompany lesions. Acral lesions on chilblains tend to be more discrete, and in the acute phase there are no pallors. In addition, although warmed, chilblains lesions are more persistent than RP lesions. $^{2}$

Lupus chilblains are a chronic and rare form of cutaneous LE. Lesions are characterized by erythematous to purple plaques located in the distal extremities, and sometimes in the nose or ears. ${ }^{4}$ These lesions are induced by cold exposure or a decrease in temperature, may accompanied by mild pain, pruritus, or hyperhidrosis. Changes in pigment and scar tissue atrophy can be seen. Lupus chilblains and RP can occur together or stand-alone. ${ }^{2}$

Acrosianosis is a change in finger color or the face becomes cyanotic, persistent, symmetrical, painless, induced by exposure to cold temperatures. This condition is often accompanied by local hyperhidrosis of the hands and feet. Acrosianosis can arise as a primary or idiopathic condition, or as a secondary manifestation of other diseases. The difference between acrocyanosis from RP is that discoloration in acrocyanosis is persistent, whereas RP is reversible. There is no pallor phase in acrocyanosis, and is not accompanied by pain. ${ }^{2}$

Livedo reticularis is a vascular pattern like a violet-colored web on the skin that is become pale with pressure. This condition is mainly suffered by young women and is often found in the lower extremities. Livedo reticularis can be a normal physiological condition that 
ISSN 25980580

disappears when warmed up, or it can also be a marker of hypercoagulable abnormalities. Livedo reticularis and RP are both associated with cyanotic discoloration due to cold exposure, and disappear when warmed. The difference is at its predilection, livedo reticularis especially attack the skin in the proximal extremities such as the thighs and knees, while the RP is more common in the acral region. ${ }^{2}$

Erythromelalgia is a condition in the form of an attack of burning or stabbing pain in the skin of the extremities induced by warm temperatures in the range of $32-36^{\circ} \mathrm{C}$. Both RP and erythromelalgia are conditions due to sensitivity to temperature, often accompanied by pain, especially regarding the hands and feet although it is more common in the lower extremities, and the present of erythematous phase. Erythromelalgia and RP, but differ in several respects. First, the ischemic phase in RP is not found in erythromelalgia. Erythromelalgia also improves with elevation of the extremities, and by cooling. ${ }^{4}$ 


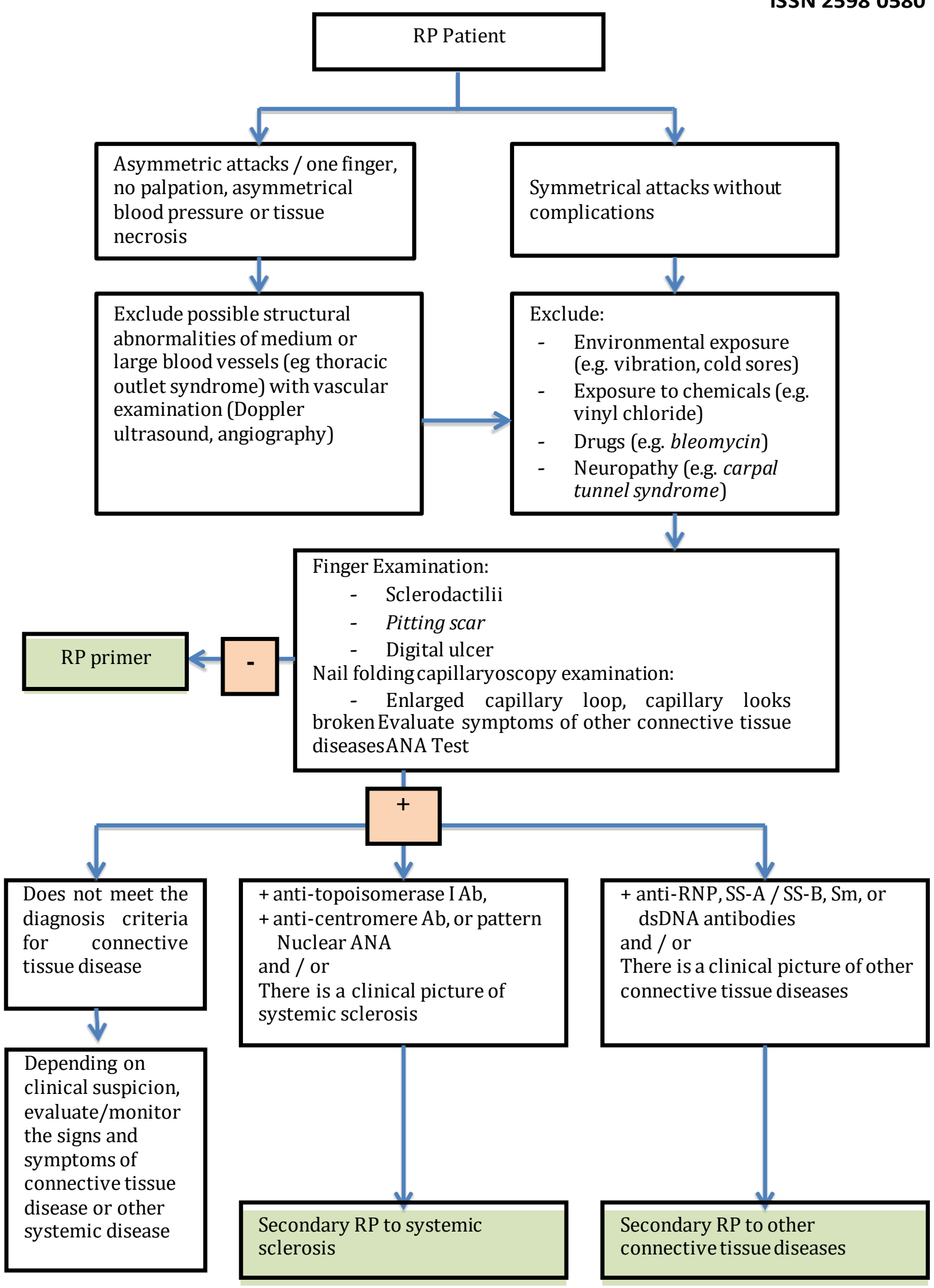

Figure 7. Chart of patient evaluation of Raynaud's phenomenon ${ }^{1}$ 


\section{Management}

ISSN 25980580

The management of RP depends on the frequency, attacks severity, and ischemic complications. In secondary RP, treatment according to the underlying disease, discontinuation of the drug causing vasospasm, or work modification is needed. Drugs are generally given to patients with prolonged attacks or who fail to be treated with conservative measures. If there is a state of severe ischemia or a finger ulcer that is resistant, and therapy with a vasodilator is not able to improve blood flow, then surgery such as digital sympathectomy is necessary. ${ }^{9}$

\section{General Management}

General or conservative management for RP consists of educating patients to change lifestyles, specifically avoiding cold exposure, stopping smoking, stress modification, and education so that patients do not feel anxious. ${ }^{10}$

Avoiding cold is the most effective therapy, and is then key in successful attacks managing in all patients. ${ }^{2}$ Local and systemic warmth is effective for increasing skin blood flow. The use of gloves, soaking and rubbing hands in warm water, or using a hair dryer can help prevent attacks or speed recovery. Generally ischemic attacks still last 15-20 minutes after warming. Systemic warm up can be done by keeping the whole body warm with loose padded clothing, gloves, and head coverings; keep the room temperature several degrees higher than normal; avoid rapidly changing temperatures, such as rushing to an air-conditioned area; limiting the time outdoors in winter; and avoid cold and windy conditions..$^{9,10}$

Smoking habits must be stopped, and other people's cigarette smoke should be avoided, because nicotine induces skin vasoconstriction. ${ }^{9}$

Stress modification, for example with counseling and relaxation training, and social support are important aspects of RP. This is because stress modification can minimize vasoconstriction due to hyperactivity of the sympathetic nervous system. ${ }^{9,10}$

Pain control is an important part of therapy, because pain for example in ulcers can cause additional vasospasm and aggravate ischemia. ${ }^{9}$

\section{Drugs}

Some classes of drugs that can be used for RP are, (1) calcium channel blockers (CCB), (2) phosphodiesterase type 5 inhibitors (PDE-5), (3) topical nitrates, (4) angiotensin II-receptor blockers (ARBs), (5) selective serotonin reuptake inhibitors (SSRIs), (6) anti-platelets, (7) prostanoids, (8) botulinum toxin type A (BTX-A), and (9) endothelin inhibitors 1. In general, 
ISSN 25980580

therapeutic responses better in primary RP patients. This is presumably because in secondary RP there has been permanent structural damage. ${ }^{9}$ Guidelines for the use of RP drugs can be seen in figure 14.

1) Calcium Channel Blockers

Calcium channel blockers are an effective first choice drug for treating RP. It is based on, calcium channels allow calcium activators to enter smooth muscle cells and initiate vasoconstriction. ${ }^{2}$

Calcium channel blockers of dihydropyridine, especially nifedipine, are the most commonly used because they are less cardioselective. Nifedipine at a dose of 10-20 mg 3-4x/day can reduce the severity and frequency of attacks. Long-acting nifedipine preparations that are at doses up to $90 \mathrm{mg} /$ day are better tolerated but may be less effective in the acute phase. ${ }^{2}$ Diltiazem is a benzothiazepine CCB that can be used if nifedipine is ineffective or not well tolerated, with a dose of $60 \mathrm{mg}$ $3 \mathrm{x} /$ day or $4 \mathrm{x} /$ day. Side effects of CCB include fluid retention, light dizziness, and heartburn. ${ }^{9}$

2) Phosphodiesterase type 5 inhibitors (PDE-5)

Phosphodiesterase is a group of enzymes that help regulate intracellular nucleotide cycle degradation. Phosphodiesterase type 5 degrades the guanosine monophosphate (GMP) cycle, which is the main mechanism for NO to cause vasodilation. PDE-5 inhibitors strengthen the vasodilator effect of NO, so this drug can be useful in the treatment of RP. ${ }^{7}$ This drug can be used single or in combination with CCB. ${ }^{10}$

3) Topical nitrates

Nowadays, the most widely used approach to handling resistant RP cases is drugs that can mimic or strengthen vasodilators and protective activity by endothelium-derived nitrite oxide. Topical nitrate, also called transdermal nitrate, which is available in patches, creams, gels and ointments, has been reported to reduce the frequency and severity of vasospastic attacks in primary and secondary RP patients without serious side effects. Nitric oxide causes dilation by stimulating guanylate cyclase and increasing the GMP cycle, which is then degraded by the enzyme PDE. ${ }^{18}$ This drug, can be used singly or in combination with CCB. ${ }^{10}$ 
4) Angiotensin II-Receptor Blockers (ARBs)

Angiotensin II-receptor blockers are drugs used for hypertension and other conditions that respond to angiotensin II blockade. The group of ARBs drugs most often used for the treatment of RP is losartan at a dose of $50 \mathrm{mg} / \mathrm{day}^{2}$

5) Selective Serotonin Reuptake Inhibitors (SSRIs)

There are several studies evaluating the use of SSRIs as RP's therapy. Selective serotonin reuptake inhibitors inhibit the reuptake of large amounts of serotonin in platelets. Digital vasodilation is likely to be partly mediated through the CNS blockade of serotonin reuptake by SSRI. ${ }^{12}$

Selective serotonin reuptake inhibitors are antidepressants and antianxiety, so they can be useful for RP patients with anxiety disorders. The most commonly used SSRI class is fluoxetine at a dose of $20 \mathrm{mg} /$ day. $^{2}$

6) Anti-platelets

Anti-platelet drugs on RP specifically used for the treatment of ulcers on the fingers of systemic scleroderma patients. Anti-platelets are mainly used in patients who have a coagulation defect as an underlying disease. ${ }^{12}$

Anti-platelet therapy with a dose between $75-81 \mathrm{mg} /$ day can be considered during an acute ischemic attack. This therapy is best used on secondary RP caused by emboli or vascular blockage process that occurs in thrombosis. ${ }^{12}$

7) Prostanoids

Prostanoids, also called prostacyclin or epoprostenol, are powerful vasodilators that act on pulmonary and systemic vascularization. This drug has many biological effects, including inhibiting smooth muscle proliferation, endothelial cell protection, and inhibiting platelet aggregation. Intravenous preparations are the form most often used for the treatment of severe secondary RP especially due to systemic sclerosis. ${ }^{2}$

8) Botulinum Toxin Type A (BTX-A)

Local injection of BTX-A on the RP can reduce pain, improve blood flow, and increase finger temperature, thereby contributing to ulcer healing. For each hand, 50 BTX-A units are injected into the palm around the neurovascular bundle as high as the metacarpophalangeal joint. Four BTX-A units are injected around the para- 
ulnar artery and the para-radial artery, and 2 units each at the beginning of the common palmar digital artery, around the initial part of the digital palmar proper artery and the para-proper palmar digital artery. The injection is done with a 30gauge needle connected to a 1 cc syringe. ${ }^{19}$

9) Endothelin inhibitors 1

Bosentan is an endothelin-1 inhibitor that has been shown to be effective in the treatment of pulmonary hypertension due to idiopathic causes and systemic sclerosis. The similarity in abnormalities in vascular function and structure in pulmonary hypertension due to systemic sclerosis and RP stimulates clinical research to study the effectiveness of bosentan in RP. Bosentan can reduce the number of new ulcers, but does not improve healing of existing ulcers, reduce the frequency, or severity of RP attacks. ${ }^{12}$

First line for mild-moderate RP:

- Begin with sustained release dihydropyridine-class calcium-channel blockers (CCB) such as nifedipine, amlodipine, or felodipin.

- If there are side effects to CCB, other options are phosphodiesterase type 5 inhibitors (PDE-5), topical nitrates, angiotensin-receptor blockers (losartan), or selective serotonin reuptake inhibitors (SSRIs)

Second line for severe RP or ischemic digital lesions:

Combination therapy, namely:

- $\mathrm{CCB}+\mathrm{PDE}-5$ inhibitors (sildenafil / tadalafil) or CCB + topical nitrate

- Add antiplatelet medication (aspirin $81 \mathrm{mg}$ )

Third line for severe recurrent $\mathrm{RP}$ or repetitive digital ischemic lesions:

- Add prostanoids (epoprostenol or iloprost) or injection of botulinum toxin, or both

- Start therapy with an endothelin-1 inhibitor (bosentan) for scleroderma with recurrent digital ulcers

Fourth line for severe digital ischemia with the threat of gangrene or amputation:

- Drug therapy + digital sympathectomy

Figure 14. RP management chart. ${ }^{10}$ 


\section{References}

1. Bashir SJ, Chew A. Cutaneous Reaction to Cold and Heat. In: Griffiths C, Barker J, Bleiker T, Chalmers R, Creamer D editors. Rook's Textbook of Dermatology $9^{\text {th }}$ ed. New York: John Wiley and Sons; 2016. p.125.1-10.

2. Wigley NM, Flavahan NA. Raynaud's Phenomenon. N Engl J Med. 2016; 375:556-65.

3. Klippel JH. Raynaud Phenomenon. In: Goldsmith LA, Katz SI, Gilchrest BA, Paller AS, Jeffell DJ, Wolff K editors. Fitzpatrick's Dermatology and General Medicine $8^{\text {th }}$ ed. New York: McGraw-Hill; 2012. p.2065-71.

4. Wigley FM, Herrick AL, Flavahan NA. Raynaud's Phenomenon: a Guide to Pathogenesis and Treatment. New York: Springer; 2015.

5. Maricq HR, Carpentier PH, Weinrich MC et al. Geographic variation in the prevalence of Raynaud's phenomenon: a 5 region comparison. J Rheumatol. 1997; 24(5):879-89.

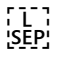

6. Garner R, Kumari R, Lanyon P, et al. Prevalence, risk factors and associations of primary Raynaud's phenomenon: systematic review and meta-analysis of observational studies. BMJ Open 2015; 5:e006389. [Cited 2018 Aug. 2]. Available from: https://bmjopen.bmj.com.

7. Brand FN, Larson MG, Kannel WB, McGuirk JM. The occurrence of Raynaud's phenomenon in a general population: The Framingham Study. Vasc. Med. 1997; 2: 296-301.

8. Grader-Beck T, Wigley FM. išpepiRaynaud's Phenomenon in Mixed Connective Tissue Disease. Rheum Dis Clin N Am 31. 2005; 31:465-81.

9. Herrick, A. L. The pathogenesis, diagnosis and treatment of Raynaud phenomenon. Nat. Rev. Rheumatol. 2012; 8:469-79.

10. Merkel PA, Herlyn K, Martin RW, Anderson JJ, Mayes MD, Bell P, et al. Measuring disease activity and functional status in patients with scleroderma and Raynaud's phenomenon. Arthritis rheumatol. 2002; 46:2410-20.

11. Connolly MK. Systemic Sclerosis (Scleroderma) and Related Disorders. In: Bolognia JL, Jorizzo JL, Schaffer JV editors. Bolognia Dermatology $3^{\text {rd }}$ ed. New York: Elsevier; 2012. p.647-51. 
12. Bakst R, Merola JF, Franks AG, Sanchez M. Raynaud's phenomenon: Pathogenesis and management. J Am Acad Dermatol. 2008; 59:633-53.

13. Fraenkel L. Raynaud's phenomenon: epidemiology and risk factors. Curr Rheumatol

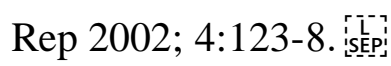

14. Chatterjee S. Raynaud phenomenon causing lingual pallor and dysarthria. Can. Med. Assoc. J. 2016; 188(15):E396. [Cited 2018 Aug. 4]. Available from: https://www.ncbi.nlm.nih.gov.

15. Prete M, Fatone MC, Favoino E, Perosa F. Raynaud's phenomenon: From molecular pathogenesis to therapy. Autoimmun Rev. 2014; 12:655-67.

16. Hartmann P, Mohokum M, Schlattmann P. The Association of Raynaud Syndrome With Thromboangiitis Obliterans-A Meta-Analysis. Angiology. 2012;63(4):315-9.

17. WHO guidelines on drawing blood: best practice in phlebotomy. Geneva: WHO, 2010.

18. Curtis P, Schwager Z, Cobos G, Lo Sicco K, Franks AG. A systematic review and metaanalysis of the effects of topical nitrates in the treatment of primary and secondary Raynaud's phenomenon. J Am Acad Dermatol. 2018; 1-12.

19. Zhang X, Hu Y, Nie Z, Song Y, Pan Y, Liu Y, et al. Treatment of Raynaud's phenomenon with botulinum toxin type A. Neurol sci. 2015;1-7. 